\title{
Effect of Hyperglycemia on Antitumor Activity and Survival in Tumor-bearing Mice Receiving Oxaliplatin and Fluorouracil
}

\author{
MAI IKEMURA ${ }^{1}$ and TOHRU HASHIDA ${ }^{2}$ \\ ${ }^{1}$ Department of Clinical Pharmacy, Faculty of Pharmaceutical Sciences, Kobe Gakuin University, Kobe, Japan; \\ ${ }^{2}$ Department of Pharmacy, Kobe City Medical Center General Hospital, Kobe, Japan
}

\begin{abstract}
Background/Aim: Diabetic patients are at a higher risk of carcinogenesis and death from cancer, including colorectal cancer, than healthy individuals. The efficacy of cancer chemotherapy in the diabetic condition remains unclear. In this study, we investigated the efficacy of anticancer agents oxaliplatin and fluorouracil in streptozotocin (STZ)-treated hyperglycemic mice. Materials and Methods: Starting 7 days after transplantation of colonic adenocarcinoma colon-26 cells, STZ-treated and control mice were intraperitoneally administered oxaliplatin, fluorouracil, and levofolinate (FOLFOX) every 2 weeks. Results: Tumor growth was delayed in STZ-treated mice compared to control mice. The tumor volume was significantly smaller after treatment with FOLFOX in control mice, but not in STZ-treated mice. Despite delayed tumor growth, and regardless of whether they received treatment, survival was shorter in STZ-treated mice than in control mice. Conclusion: Cancer chemotherapy with oxaliplatin and fluorouracil was less effective and survival was shorter in hyperglycemia.
\end{abstract}

Diabetic patients have been reported to have a higher risk of carcinogenesis and mortality from various types of cancer, including colorectal cancer, than healthy individuals (1-3). However, the mechanisms underlying this relationship have not been elucidated in detail. As one of the possible mechanisms, we previously reported that tumor metastasis was accelerated in streptozotocin (STZ)-treated hyperglycemic mice (4).

Chemotherapy is a major therapeutic approach for various types of cancers. Some reports have indicated that in diabetic patients with various kinds of cancers, the risk of

Correspondence to: Mai Ikemura, Department of Clinical Pharmacy, Faculty of Pharmaceutical Sciences, Kobe Gakuin University, 1-1-3 Minatojima, Chuo-ku, Kobe, Hyogo, Japan. Email: mai.ikemura@pharm.kobegakuin.ac.jp

Key Words: Diabetes, hyperglycemia, cancer chemotherapy, oxaliplatin, fluorouracil, colorectal cancer. chemotherapy-related toxicity was increased and overall survival was shortened compared to that in non-diabetic patients (5-7). These results suggested that cancer chemotherapy was less effective and less safe in diabetic patients than in non-diabetic patients. However, the causes or mechanisms involved remain unclear and there is limited information regarding therapeutic strategies for cancer in diabetic patients $(8,9)$.

In this study, we focused on the influence of the diabetic condition on the efficacy of cancer chemotherapy. Some reports have evaluated the effect of glucose levels on the cytotoxicity of anticancer agents in vitro (10-12), but few reports focused on efficacy with respect to both tumor volume and survival in diabetes in vivo. Although anticancer agents are usually used in combination as part of established regimens, there are very few reports that evaluated these agents using clinical regimen-based treatment in animals. Colorectal cancer is known to have a higher incidence and mortality in diabetic patients $(1-3,13)$. It is also as a cancer type in which chemotherapy is frequently used for patients undergoing surgery as neoadjuvant or adjuvant therapy and for those with advanced-stage or recurrent cancer. Therefore, elucidation of the efficacy and safety of cancer chemotherapy in colorectal cancer in those who are diabetic would contribute to the decision-making strategy regarding treatment in diabetic patients. In this study, we investigated antitumor activity in and survival of STZ-treated insulin-deficient hyperglycemic mice who received oxaliplatin, fluorouracil, and levofolinate, which are used in the FOLFOX chemotherapy regimen that is frequently used for colorectal cancer therapy.

\section{Materials and Methods}

Chemicals. STZ was obtained from Sigma-Aldrich (St. Louis, MO, USA). Phosphate-buffered saline (PBS) and Hanks' balanced salt solution (HBSS) were obtained from Nissui Pharmaceutical (Tokyo, Japan). RPMI-1640, penicillin-streptomycin, glutamine, D-glucose, and D-mannitol were obtained from Nacalai Tesque (Kyoto, Japan). Tumor cells. Murine colon adenocarcinoma colon-26 cells, obtained from the Cell Engineering Division, RIKEN BioResource Center (Tsukuba, Japan), were grown in RPMI-1640 supplemented with 
a

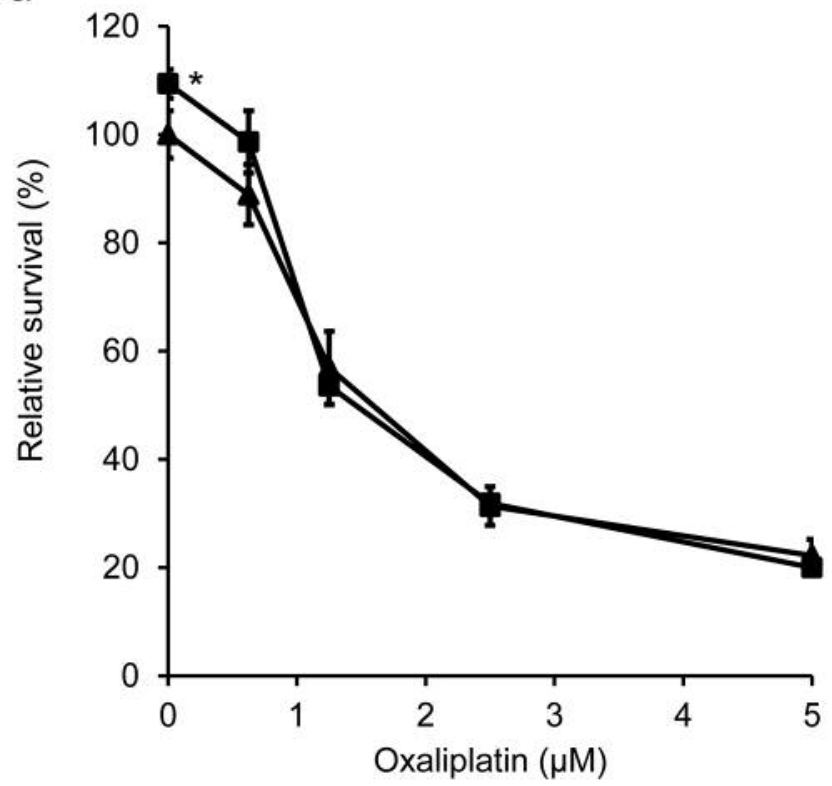

b

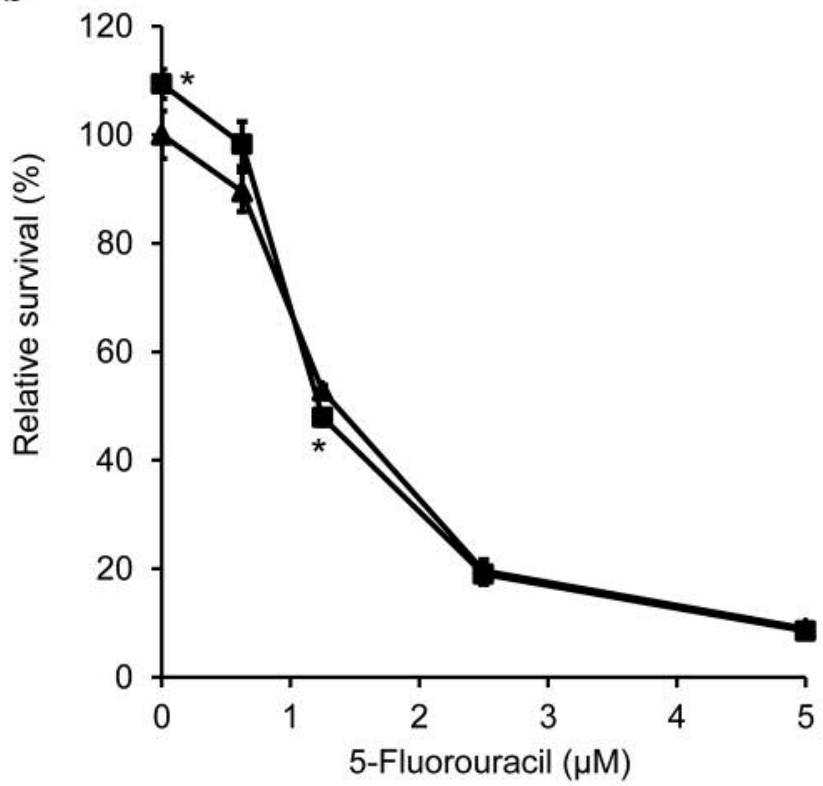

Figure 1. Cytotoxic effect of oxaliplatin (a) and 5-fluorouracil (b) under normal and high-glucose conditions in vitro. Colon-26 cells were treated with oxaliplatin or fluorouracil under normal (5 mM glucose $+20 \mathrm{mM}$ mannitol, triangle) or high glucose conditions (25 $\mathrm{mM}$ glucose, square). The number of cells was determined $72 \mathrm{~h}$ after treatment. Results are expressed as the mean $\pm S D(n=3) . *$ Statistically significant difference compared to normal glucose conditions at $p<0.05$.

$10 \%$ heat-inactivated fetal bovine serum (FBS), $0.15 \% \mathrm{NaHCO}_{3}$, $100 \mathrm{U} / \mathrm{ml}$ penicillin, and $100 \mu \mathrm{g} / \mathrm{ml}$ streptomycin at $37^{\circ} \mathrm{C}$ in humidified air containing $5 \% \mathrm{CO}_{2}$.

Cytotoxic effect of anticancer agents under normal and highglucose conditions in vitro. Colon-26 cells were seeded on 96-well plates at a density of $5 \times 10^{3}$ cells per well in medium containing $1 \%$ FBS and $5 \mathrm{mM}$ glucose. After $24 \mathrm{~h}$ of incubation, cells were treated with $0,0.625,1.25,2.5$, or $5 \mu \mathrm{M}$ oxaliplatin (Yakult Honsha Co., Ltd., Tokyo, Japan) or $0,0.625,1.25,2.5$, or $5 \mu \mathrm{M}$ fluorouracil (Kyowa Hakko Kirin Co., Ltd., Tokyo, Japan) in medium containing either $1 \%$ FBS, $5 \mathrm{mM}$ glucose, and $20 \mathrm{mM}$ mannitol (normal glucose), or $25 \mathrm{mM}$ glucose (high glucose). Seventy-two hours after treatment, the number of viable cells was determined using Cell Counting Kit-8 (Dojindo, Kumamoto, Japan).

Animals. BALB/c mice (male, 8 weeks old) were purchased from the Shizuoka Agricultural Cooperative Association for Laboratory Animals (Shizuoka, Japan). Animals were maintained in a temperature- and humidity-controlled room with $12 \mathrm{~h} \mathrm{light/dark}$ cycle and allowed access to food and water ad libitum. All animal experiments were approved by the Animal Experimentation Committee of Kobe Gakuin University (No. 16-33).

Production of hyperglycemic model mice and transplantation of tumor cells. Mice received an intraperitoneal injection of STZ dissolved in sodium citrate buffer $(\mathrm{pH} 4.5)$ at a dose of $150 \mathrm{mg} / \mathrm{kg}$ body weight or buffer alone. One week after the injection, blood glucose levels were determined using a LIFE CHECK (Eidia Co., Ltd, Tokyo, Japan) or Stat Strip XP3 (Nipro, Osaka, Japan). Mice whose glucose levels were greater than $300 \mathrm{mg} / \mathrm{dl}$ were used as hyperglycemic models. Four weeks after the onset of the hyperglycemic state, each mouse received a subcutaneous injection of $1 \times 10^{5}$ colon- 26 cells in $0.1 \mathrm{ml}$ HBSS.

Administration of anticancer agents and evaluation of tumor growth and survival. Colon-26 cell-bearing mice were divided into four groups: saline-receiving control mice, FOLFOX (oxaliplatin, fluorouracil, and levofolinate)-receiving control mice, saline-receiving STZ-treated mice, and FOLFOX-receiving STZ-treated mice. Seven days after tumor transplantation, mice received FOLFOX or saline. The administration schedule was determined based on the modified FOLFOX6 chemotherapy regimen used in clinical settings. Dosages were determined by translation from humans to animals (14) and modified from a previous report (15) and preliminary experiments that investigated fatal doses. Oxaliplatin $(6 \mathrm{mg} / \mathrm{kg})$ was administered, and $2 \mathrm{~h}$ later, calcium levofolinate (Nippon Kayaku Co., Ltd., Tokyo, Japan) $(50 \mathrm{mg} / \mathrm{kg})$ dissolved in saline and fluorouracil $(50 \mathrm{mg} / \mathrm{kg})$ were administered intraperitoneally. Dosages are stated as quantities of active agents. Control mice received same volume of saline. These administrations were repeated every two weeks.

The bodyweight of the mice was measured $0,1,3,7,10,14,15$, 17 , and 21 days after the first administration of FOLFOX or saline. Tumor volume was measured using calipers at 7, 10, 14, 17, and 21 days after the first administration. The volume and tumor-inhibitory rate were calculated as follows: volume $\left(\mathrm{mm}^{3}\right)=$ long diameter $(\mathrm{mm}) \times\left[\right.$ short diameter $\left.(\mathrm{mm})^{2}\right] / 2$; tumor-inhibitory rate $(\%)=[1-$ (mean tumor volume of FOLFOX-receiving mice)/(mean tumor volume of saline-receiving mice) $] \times 100$. In addition, survival of the mice in each group was monitored until 35 days after the first administration of FOLFOX or saline. 


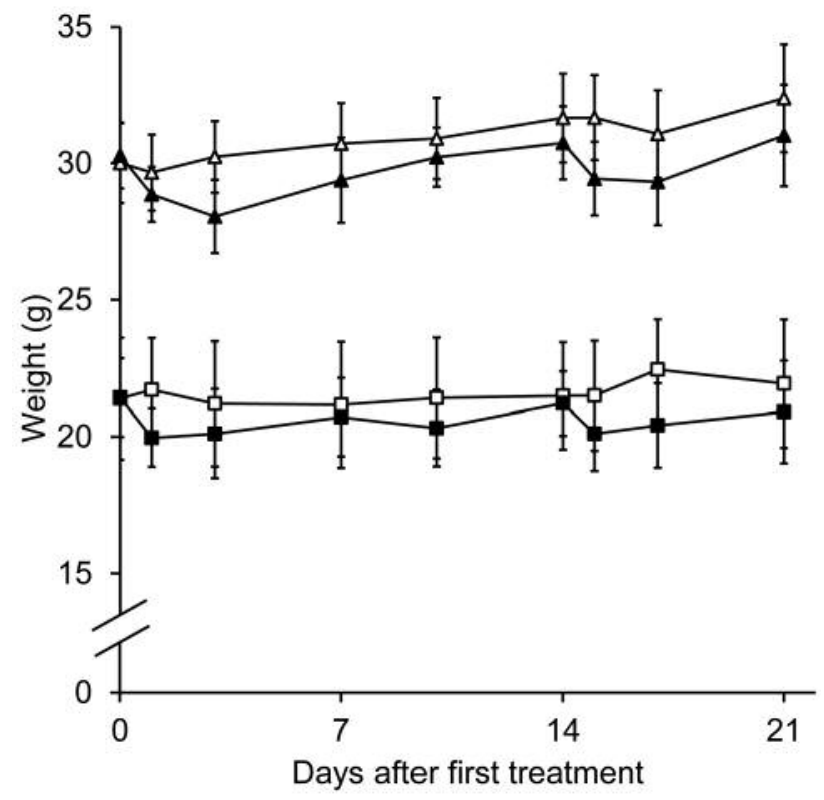

Figure 2. Bodyweight change in control (triangle) and streptozotocin (STZ)-treated (square) mice receiving a subcutaneous transplantation of colon-26 cells. Seven and 21 days after tumor transplantation (day 0 and 14), saline (open symbols) or FOLFOX (closed symbols) was administered. The bodyweight of mice was measured at $0,1,3,7,10$, $14,15,17$, and 21 days after the first administration. Results are expressed as the mean $\pm S D$ of eight mice in each group; note two FOLFOX-receiving STZ-treated mice died at 18 and 20 days.

Measurement of plasma interleukin-6 (IL6) and albumin levels. Blood samples were obtained from the orbital vein of each mouse and plasma was obtained by centrifugation 35 days after the first administration of FOLFOX. Plasma IL6 and albumin levels were determined using commercial kits obtained from BioLegend, Inc. (San Diego, CA, USA) and Wako Pure Chemical Industries, Ltd. (Osaka, Japan), respectively, according to the test protocol. Samples were diluted as necessary.

Statistical analysis. Differences were evaluated statistically using the Student $t$-test (cytotoxic effect of anticancer agents), one-way ANOVA (plasma albumin level) followed by Tukey-Kramer test (body weight), Welch's test followed by Games-Howell test (tumor volume), and the Kruskal-Wallis test followed by the Steel-Dwass test (plasma IL6 level). Survival was estimated using Kaplan-Meier analysis with a log-rank test. The significance of differences was set at $p<0.05$.

\section{Results}

Cytotoxic effect of oxaliplatin and fluorouracil under normal and high-glucose conditions in vitro. To evaluate the influence of glucose itself on the anticancer activity of oxaliplatin and fluorouracil, we examined the cytotoxic effect on colon-26 cells under normal ( $5 \mathrm{mM}$ glucose plus $20 \mathrm{mM}$ mannitol) and high-glucose (25 mM glucose) conditions (Figure 1). In the absence of antitumor agents, the cell proliferative rate was

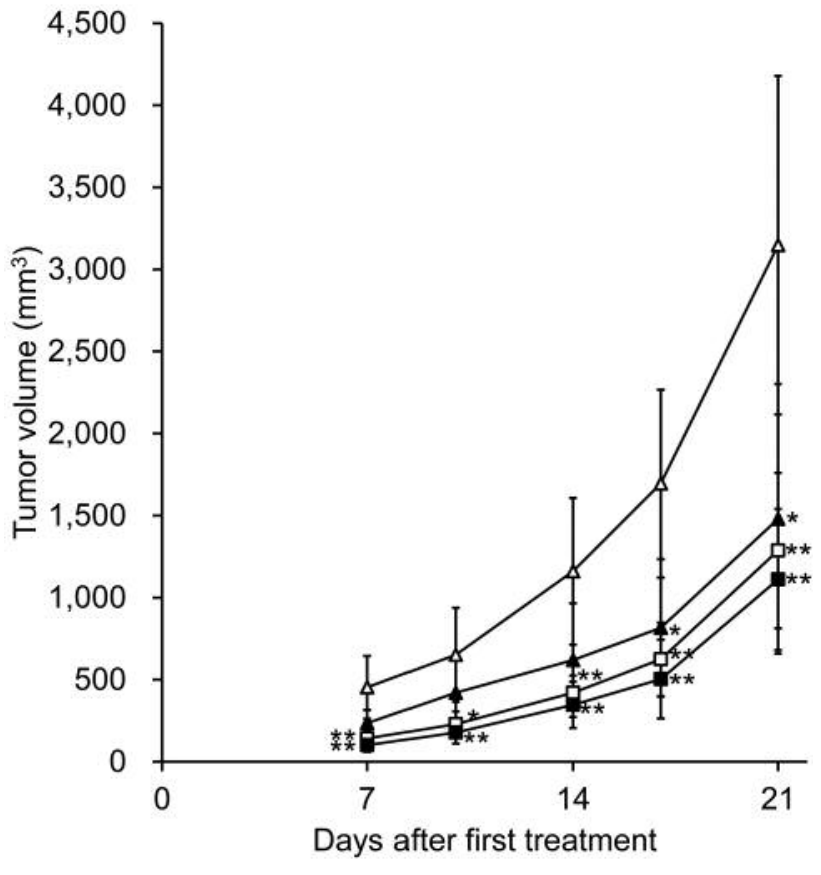

Figure 3. Tumor volume in control (triangle) and streptozotocin (STZ)treated (square) mice receiving a subcutaneous transplantation of colon26 cells. Seven and 21 days after tumor transplantation (day 0 and 14), saline (open symbols) or FOLFOX (closed symbols) was administered. Tumor volume of mice was measured at 7, 10, 14, 17, and 21 days after the first treatment administration. Results are expressed as the mean $\pm S D$ of eight mice in each group; note two FOLFOX-receiving STZ-treated mice died at 18 and 20 days. Statistically significant difference compared with saline-receiving control mice at $* p<0.05$ and $* * p<0.01$.

higher under high-glucose conditions than under normalglucose conditions. The cytotoxic effects of oxaliplatin and fluorouracil were similar under both glucose conditions.

Bodyweight changes after FOLFOX treatment in control and STZ-treated mice. Figure 2 shows the body weight changes in saline- and in FOLFOX-receiving control and STZ-treated mice. The bodyweight of STZ-treated mice was significantly lower than that of control mice during the study. FOLFOX treatment slightly reduced body weight, but the reduction was not significant in control or STZ-treated mice.

Effect of FOLFOX on tumor volume in control and STZtreated mice. Figure 3 shows the temporal change in tumor volume in saline- and in FOLFOX-receiving control and STZ-treated mice. Tumor volume was significantly smaller in STZ-treated mice than in control mice during the study. Tumor growth was significantly inhibited by FOLFOX treatment in control mice. Conversely, tumor growth was only slightly inhibited by FOLFOX in STZ-treated mice. The 
tumor-inhibitory rate after FOLFOX treatment was $48 \%$ and $28 \%$ at day $7,47 \%$ and $18 \%$ at day 14 , and $53 \%$ and $14 \%$ at day 21 , in control and STZ-treated mice, respectively.

Survival of saline- and FOLFOX-receiving control and STZtreated mice. Figure 4 shows overall survival for saline- and FOLFOX-receiving control and STZ-treated mice. Survival of STZ-treated mice was significantly shorter than that of control mice. FOLFOX prolonged survival of most of the control mice. Survival was shorter for STZ-treated mice, regardless of the treatment received.

Plasma IL6 and albumin levels in saline- and FOLFOXreceiving control and $S T Z$-treated mice. We examined plasma IL6 and albumin levels as mediators of cancer cachexia in saline- and FOLFOX-receiving control and STZ-treated mice (Figure 5). Plasma IL6 levels were significantly lower in FOLFOX-receiving control mice compared with those in saline-receiving control mice (Figure 5a). Plasma IL6 levels were also significantly lower in saline-receiving STZ-treated mice than in saline-receiving control mice. There was no significant difference in the IL6 levels in saline- and FOLFOX-receiving STZ-treated mice. Plasma albumin levels were similar among all of the groups (Figure 5b).

\section{Discussion}

Previous reports have suggested that the efficacy and safety of cancer chemotherapy are worse and overall survival is shorter in diabetic than in non-diabetic patients (5-7). However, the factors that affect the efficacy and safety of chemotherapy have not been fully elucidated. In this study, we evaluated tumor volume and survival in animals because frequent evaluation of tumor volume and comparison with subjects receiving no anticancer agents were needed, which could not be evaluated in human patients owing to ethical issues. In a previous report, survival was evaluated in chemically induced cancer models with diabetes that were treated with or without oxaliplatin (16). The present study demonstrated that survival was shorter despite slower tumor growth in STZ-treated mice. Furthermore, FOLFOX, a clinical regimen-based therapy, did not inhibit tumor growth or prolong survival in STZ-treated mice, whereas it did inhibit tumor growth and prolong survival in control mice.

In the in vitro study, cell proliferation was promoted under high-glucose conditions (Figure 1), which was consistent with previous reports $(10,11,17)$. In contrast, tumor growth was slower in STZ-treated mice than in control mice (Figure 3). STZtreated insulin-deficient mice have different characteristics from control mice, including different blood glucose and insulin levels, and tissue functions. Therefore, tumor growth was slowed in STZ-treated mice, resulting not from glucose itself, but likely from insulin level or factors induced by hyperglycemia.

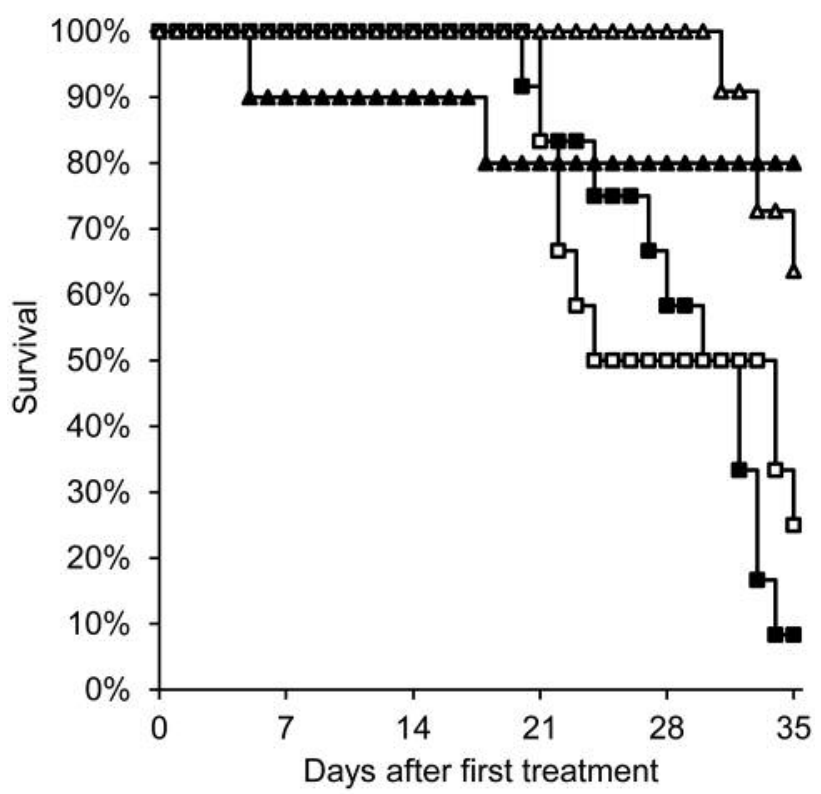

Figure 4. Survival of control (triangle) and streptozotocin (STZ)-treated (square) mice receiving a subcutaneous transplantation of colon-26 cells. Seven, 21, and 35 days after tumor transplantation (day 0, 14, and 28), saline (open symbols) or FOLFOX (closed symbols) was administered to control mice ( $n=11$, and $n=10$, respectively), and STZ-treated mice (both $n=12)$. The survival of the saline-receiving STZ-treated mice was significantly poorer than that of saline-receiving control mice $(p=0.038)$ and the survival of FOLFOX-receiving STZ-treated mice was significantly poorer than that of FOLFOX-receiving control mice $(p=0.004)$.

In STZ-treated mice, the effect of the anticancer agents on tumor volume was small (Figure 3). This may have been caused by a lower sensitivity to anticancer agents or changes in pharmacokinetics. In the in vitro study, the cytotoxic effects of oxaliplatin and fluorouracil were similar under normal and highglucose conditions (Figure 1), indicating that glucose itself did not markedly affect the antitumor activity of the agents. In this study, cells were exposed to high glucose for only $72 \mathrm{~h}$. Exposure to glucose for a longer time might change the characteristics of the cells. With respect to pharmacokinetics, STZ-treated rats have been reported to exhibit a faster total body clearance and smaller area under the plasma concentration-time curve (AUC) after fluorouracil infusion than buffer-treated rats (18). Although there are differences between the kinds of agents and the activity of metabolic enzymes due to species differences, the distribution of oxaliplatin and fluorouracil might also be altered in STZ-treated mice.

Survival was prolonged by FOLFOX in most of the control mice (Figure 4). Two of the FOLFOX-receiving control mice died at an early date, which might have been caused by the side-effects of FOLFOX and not by cancer. Conversely, survival was similar in saline- and FOLFOX-receiving STZtreated mice. Previous reports also indicated that treatment with 
a

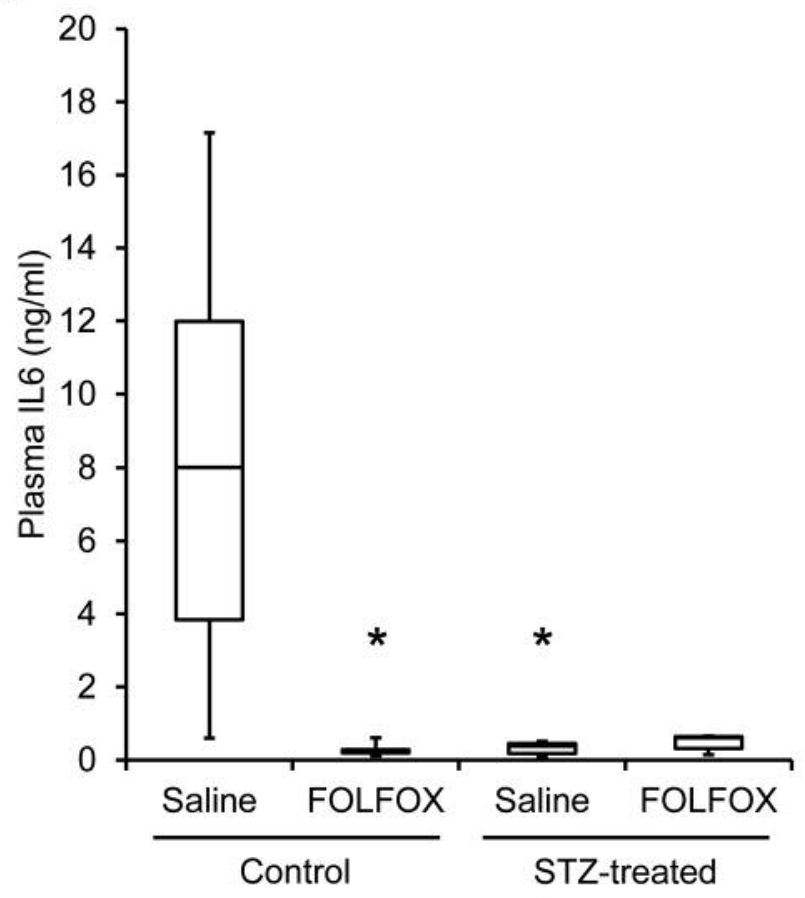

b

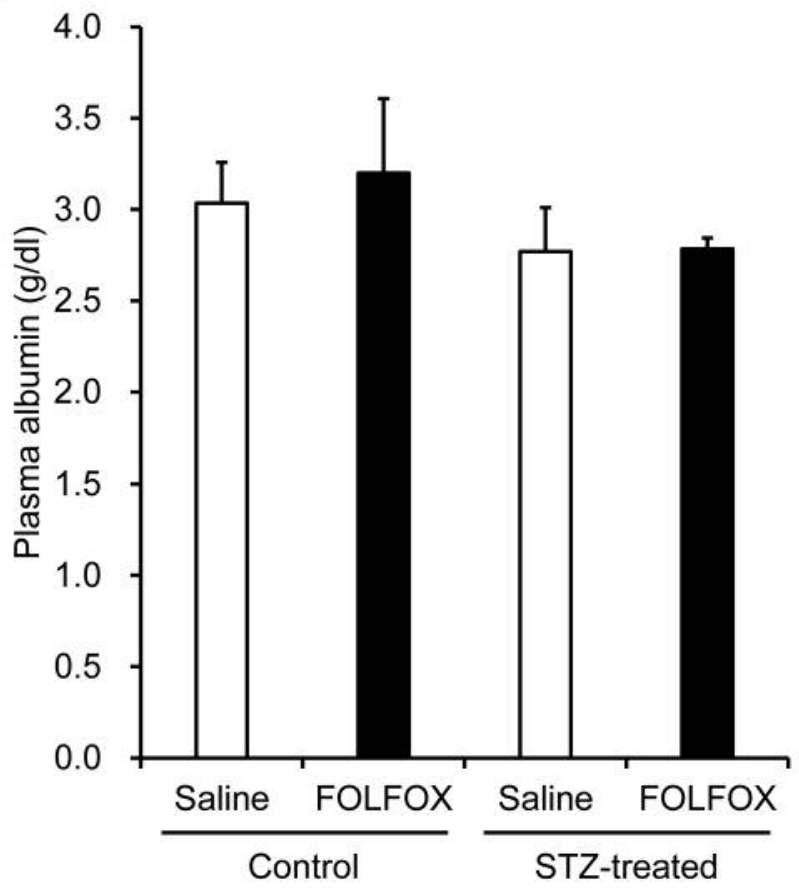

Figure 5. Cancer cachexia in mice receiving a subcutaneous transplantation of colon-26 cells. Seven, 21 , and 35 days after tumor transplantation (day 0, 14, and 28), saline or FOLFOX was administered and blood samples were collected at 35 days. a: Plasma interleukin-6 (IL6) levels. Lines in the center of the box indicate the median and the upper and lower lines indicate the 75th and 25th percentile, respectively., *Statistically significant difference compared with saline-receiving control mice at $p<0.05$. b: Plasma albumin levels. Results are expressed as mean $+S D$ of 3-6 mice.

antitumor agents did not prolong survival in diabetic mice $(9$, 16). Interestingly, we found that survival was shorter despite slower tumor growth in STZ-treated mice, regardless of whether they received saline or FOLFOX treatment, compared to that for saline-receiving control mice (Figures 3 and 4). Death might have been caused by the presence of cancer in both saline- and FOLFOX-receiving STZ-treated mice because tumor volume was also similar. To elucidate the details, we evaluated cancer cachexia in control and STZ-treated mice. IL6 is a mediator of cancer cachexia and is detectable in the plasma of colon-26-bearing mice $(19,20)$. In this study, plasma IL6 levels were the highest in saline-receiving control mice (Figure 5a). The IL6 levels approximately reflected the tumor volume and were not as high in STZ-treated mice as in control mice. This suggests that shorter survival despite a smaller primary tumor volume was not caused by cancer cachexia under hyperglycemia. Our previous report indicated that tumor metastasis after intravenous transplantation of melanoma cells was accelerated in STZ-treated mice (4). Even in the present study, acceleration of tumor metastasis in STZ-treated mice might have been a cause of their shorter survival. However, the process of tumor metastasis would have been different because of the different route of transplantation and cell type. Therefore, the involvement of tumor metastasis is not clear.
Although this study was performed under conditions as similar to clinical conditions as possible, there were some differences and limitations. Firstly, there were no preventive strategies against the toxicities of chemotherapy. Although oxaliplatin, fluorouracil, and levofolinate were administered, agents for supportive care were not. Side-effects were not managed because there was no monitoring, routine examination, or 'patient complaints'. Therefore, the dosage of anticancer agents was not adjusted according to side-effects. Secondly, agents were administered intraperitoneally in this study, whereas an intravenous drip is used in clinical settings. Although plasma concentration increases gradually after administration by both routes, the difference in these routes might affect pharmacokinetics, that might affect antitumor activity. Thirdly, cancer cells were transplanted subcutaneously in this study, whereas, clinically, they are generated spontaneously. The results might depend on the transplanted cell types or procedures for the generation of tumors. In this study, in order to evaluate tumor growth, we used mice that received a subcutaneous transplantation of cancer cells. Fourthly, we used an insulin-deficient hyperglycemic mouse model. Hyperglycemia is usually controlled by therapeutic agents in diabetic patients, but was not controlled in this study. One of the indices of glycemic control, the hemoglobin A1c level, has been 
reported to be associated with the incidence and mortality of some types of cancer $(21,22)$. Findings obtained in this study were consistent with those reports.

In conclusion, our investigation demonstrated that the antitumor activity of oxaliplatin and fluorouracil was lower in STZ-treated hyperglycemic mice and their overall survival was shorter regardless of treatment. This effect was not induced by glucose itself or by cancer cachexia. In clinical settings, the therapeutic effect of anticancer agents may be less under hyperglycemic conditions compared with that under normoglycemic condition when the tumor size is similar at diagnosis or first therapy administration. These results suggest that cancer chemotherapy with oxaliplatin and fluorouracil provides a lesser therapeutic effect and survival advantage in hyperglycemia. Further studies are needed to clarify the mechanisms leading to a shorter survival despite slower tumor growth in hyperglycemia and elucidate the influence of glucose levels.

\section{Acknowledgements}

This work was supported in part by JSPS KAKENHI (Grant number 15K18940) and the Suzuken Memorial Foundation.

\section{References}

1 Barone BB, Yeh HC, Snyder CF, Peairs KS, Stein KB, Derr RL, Wolff AC and Brancati FL: Long-term all-cause mortality in cancer patients with preexisting diabetes mellitus: a systematic review and meta-analysis. JAMA 300: 2754-2764, 2008.

2 Vigneri P, Frasca F, Sciacca L, Pandini G and Vigneri R: Diabetes and cancer. Endocr Relat Cancer 16: 1103-1123, 2009.

3 Sasazuki S, Charvat H, Hara A, Wakai K, Nagata C, Nakamura K, Tsuji I, Sugawara Y, Tamakoshi A, Matsuo K, Oze I, Mizoue $\mathrm{T}$, Tanaka K, Inoue M, Tsugane S and for the Research Group for the Development and Evaluation of Cancer Prevention Strategies in Japan: Diabetes mellitus and cancer risk: Pooled analysis of eight cohort studies in Japan. Cancer Sci 104: 1499-1507, 2013.

4 Ikemura M, Nishikawa M, Kusamori K, Fukuoka M, Yamashita $\mathrm{F}$ and Hashida M: Pivotal role of oxidative stress in tumor metastasis under diabetic conditions in mice. J Control Release 170: 191-197, 2013.

5 Srokowski TP, Fang S, Hortobagyi GN and Giordano SH: Impact of diabetes mellitus on complications and outcomes of adjuvant chemotherapy in older patients with breast cancer. J Clin Oncol 27: 2170-2176, 2009.

6 Ottaiano A, Nappi A, Tafuto S, Nasti G, De Divitiis C, Romano C, Cassata A, Casaretti R, Silvestro L, Avallone A, Capuozzo M, Capozzi M, Maiolino P, Quagliariello V, Scala S and Iaffaioli VR: Diabetes and body mass index are associated with neuropathy and prognosis in colon cancer patients treated with capecitabine and oxaliplatin adjuvant chemotherapy. Oncology 90: 36-42, 2016.

7 Meyerhardt JA, Catalano PJ, Haller DG, Mayer RJ, Macdonald JS, Benson $\mathrm{AB}$ 3rd and Fuchs CS: Impact of diabetes mellitus on outcomes in patients with colon cancer. J Clin Oncol 21: 433-440, 2003.
8 Richardson LC and Pollack LA: Therapy insight: influence of type 2 diabetes on the development, treatment and outcomes of cancer. Nat Clin Pract Oncol 2: 48-53, 2005.

9 de Silva Faria MC, Santos NA, Carvalho Rodrigues MA, Rodrigues JL, Barbosa Junior F and Santos AC: Effect of diabetes on biodistribution, nephrotoxicity and antitumor activity of cisplatin in mice. Chem Biol Interact 229: 119-131, 2015.

10 Pandey V, Chaube B and Bhat MK: Hyperglycemia regulates MDR-1, drug accumulation and ROS levels causing increased toxicity of carboplatin and 5-fluorouracil in MCF-7 cells. J Cell Biochem 112: 2942-2952, 2011.

11 Ma YS, Yang IP, Tsai HL, Huang CW, Juo SH and Wang JY: High glucose modulates antiproliferative effect and cytotoxicity of 5-fluorouracil in human colon cancer cells. DNA Cell Biol 33: 64-72, 2014.

12 Volkova E, Robinson BA, Willis J, Currie MJ and Dachs G: Marginal effects of glucose, insulin and insulin-like growth factor on chemotherapy response in endothelial and colorectal cancer cells. Oncol Lett 7: 311-320, 2014.

13 Mills KT, Bellows CF, Hoffman AE, Kelly TN and Gagliardi G: Diabetes mellitus and colorectal cancer prognosis: a metaanalysis. Dis Colon Rectum 56: 1304-1319, 2013.

14 Reagan-Shaw S, Nihal M and Ahmad N: Dose translation from animal to human studies revisited. FASEB J 22: 659-661, 2008.

15 Robinson SM, Mann DA, Manas DM, Oakley F, Mann J and White SA: The potential contribution of tumour-related factors to the development of FOLFOX-induced sinusoidal obstruction syndrome. Brit J Cancer 109: 2396-2403, 2013.

16 Zaafar DK, Zaitone SA and Moustafa YM: Role of metformin in suppressing 1,2-dimethylhydrazine-induced colon cancer in diabetic and non-diabetic mice: effect on tumor angiogenesis and cell proliferation. PLoS One 9: e100562, 2014.

17 Feng YH, Lin CY, Huang WT, Wu CL, Fang JL and Tsao CJ: Diabetes mellitus impairs the response to intra-arterial chemotherapy in hepatocellular carcinoma. Med Oncol 28: 1080-1088, 2011.

18 Choi YH, Lee AK, Bae SK, Kim SO and Lee MG: Pharmacokinetics of 5-fluorouracil in rats with diabetes mellitus induced by streptozotocin. Biopharm Drug Dispos 26: 93-98, 2005.

19 Matsumoto T, Fujimoto-Ouchi K, Tamura S, Tanaka Y and Ishitsuka $\mathrm{H}$ : Tumour inoculation site-dependent induction of cachexia in mice bearing colon 26 carcinoma. Br J Cancer 79: 764-769, 1999.

20 Strassmann G, Fong M, Kenney JS and Jacob CO: Evidence for the involvement of interleukin 6 in experimental cancer cachexia. J Clin Invest 89: 1681-1684, 1992.

21 Hope C, Robertshaw A, Cheung KL, Idris I and English E: Systematic review or meta-analysis relationship between HbA1c and cancer in people with or without diabetes: a systematic review. Diabet Med 33: 1013-1025, 2016.

22 Goto A, Noda M, Sawada N, Kato M, Hidaka A, Mizoue T, Shimazu T, Yamaji T, Iwasaki M, Sasazuki S, Inoue M, Kadowaki T, Tsugane S and JPHC Study Group: High hemoglobin A1c levels within the non-diabetic range are associated with the risk of all cancers. Int J Cancer 138: 1741-1753, 2016.

Received July 21, 2017

Revised August 7, 2017

Accepted August 8, 2017 